\title{
Global work index by non-invasive pressure-strain loops: a novel parameter to assess left ventricular performance in the early stages of heart failure with preserved or mid-range ejection fraction after acute myocardial infarction
}

\author{
Raluca Tomoaia', ${ }^{1,2}$, Ruxandra Ștefana Beyer ${ }^{1}$, Dumitru Zdrenghea ${ }^{2,3}$, Alexandra Dădârlat- \\ Pop $^{1,2}$, Gabriel Cismaru ${ }^{2,3}$, Gabriel Gușetu ${ }^{2,3}$, Radu Roșu ${ }^{2,3}$, Adela Șerban ${ }^{1,2}$, Dana Pop ${ }^{2,3}$
}

${ }^{1}$ Heart Institute "Nicolae Stancioiu", ${ }^{2}$ 'Iuliu Hatieganu” University of Medicine and Pharmacy, ${ }^{3}$ Cardiology Department, Rehabilitation Hospital, Cluj-Napoca, Romania

\begin{abstract}
Aim: To determine the relationship between myocardial work (MW) indices derived from non-invasive pressure-strain loops (PSL) and established parameters of left ventricular (LV) performance in patients with acute myocardial infarction (AMI) and heart failure (HF) with preserved or mid-range ejection fraction (HFpEF/HFmrEF). Material and methods: Speckle-tracking echocardiography (STE) was used to determine MW indices and global longitudinal strain (GLS) in the first 24-48 hours after admission in patients with AMI, HF symptoms, NT-proBNP $>300 \mathrm{pg} / \mathrm{mL}$ and left ventricular ejection fraction (LVEF) $>40 \%$. MW was calculated by using PSL, which combine strain and non-invasive blood pressure measurement. Global MW index (GWI) was defined as the work inside the area of the PSL. Results: Forty-nine patients (mean age $68 \pm 13$ years) fulfilled the inclusion criteria. Both GWI $(1057 \pm 338 \mathrm{mmHg} \%)$ and GLS $(-10.4 \pm 3.3 \%)$ were reduced in the majority of the patients. However, a proportion of patients with abnormal GLS showed normal GWI. There was a strong inverse relationship between GWI and GLS ( $\mathrm{r}=-0.81)$. GWI demonstrated a strong relationship with LVEF ( $\mathrm{r}=0.69)$ and a moderate correlation to NT-proBNP $(\mathrm{n}=-0.29)$. NT-proBNP showed a tendency to higher values in patients with more reduced GWI (820 [590-2550] vs 707 [460-1335], $\mathrm{p}=0.17)$. Out of the diastolic dysfunction parameters, GWI showed moderate correlations to LA volume index $(\mathrm{r}-0.29), \mathrm{E} / \mathrm{A}(\mathrm{r}=-0.23)$ and $\mathrm{E} / \mathrm{e}^{\prime}(\mathrm{r}=-0.39)$, which were also significantly more impaired in patients with more reduced GWI. Conclusion: Non-invasive PSL might bring further information to LVEF and GLS in patients AMI at the early stages of HFpEF/HFmrEF, since LV performance depends on both contractile properties and variations in load in the ischemic segments.
\end{abstract}

Keywords: myocardial work; acute myocardial infarction; speckle-tracking echocardiography; heart failure with preserved ejection fraction

\section{Introduction}

Acute myocardial infarction (AMI) leads to sudden reduction in blood flow into the myocardium and immedi-

Received 14.06.2020 Accepted 25.09.2020

Med Ultrason

2021, Vol. 23, No 1, 62-69

Corresponding author: Raluca Tomoaia

"Niculae Stancioiu" Heart Institute

19-21, Motilor street,

400001, Cluj-Napoca, Romania

Phone: +40264597256

E-mail: raluca.tomoaia@gmail.com ate impairment of the left ventricular (LV) performance. In ischemic segments, the reduction in systolic function depends on both contractile properties and variations in load. In patients with heart failure (HF), the adaptive response to increased afterload is impaired $[1,2]$. In the early stages of HF, when LV ejection fraction (LVEF) is still preserved, increased LV end-diastolic pressure leads to increased mechanical stress in the left atrium (LA). If increased afterload persists over time, LV needs to generate greater force in order to preserve the stroke volume by increasing the consumed energy and leading to remodeling $[3,4]$. LVEF is still the most frequently used pa- 
rameter in the evaluation of systolic function in patients with AMI, despite its limitations [5]. Global longitudinal strain (GLS) has proven its reliability in the evaluation of cardiac mechanics, by adding information to LV performance $[6,7]$ since it has ability in detecting more subtle changes when LVEF is normal [8]. However, GLS is still load dependent and an increase in afterload has been demonstrated to decrease strain, leading to misinterpretation of the true contractile function $[9,10]$.

Recently, it was demonstrated that LV strain and afterload could be simultaneously assessed. Russel et al presented a method for calculating non-invasive myocardial work (MW) by speckle-tracking echocardiography (STE) and estimation of left ventricular (LV) pressure from brachial cuff pressure. MW derived from pressure-strain loops (PSL) is included in available echocardiographic software and has been demonstrated to provide excellent agreement between measured and estimated pressure-loops $[11,12]$. Due to the added value of LV pressure, MW brings further information to LVEF and GLS in the quantification of LV performance. Some studies have shown that MW might be a better indicator of LV systolic performance in patients with dilated cardiomyopathy, non-ST-segment-elevation AMI (NSTEMI), HF and arterial hypertension $[9,13,14]$. However, the importance of MW in patients with HF with preserved or mid-range LVEF (HFpEF/ HFmrEF) occurring in the setting of AMI has not been previously studied.

The aim of this study was to determine the relationship between MW indices, established echocardiographic parameters of LV dysfunction (LVEF, GLS and parameters used in the quantification of diastolic dysfunction) and NT-proBNP in patients with HFpEF or HFmrEF occurring in the setting of AMI.

\section{Material and methods}

\section{Study population}

This was a single center cross-sectional study. After approval from the Human Ethics Committeeand after written informed consent was obtained from all patients,49 subjects with AMI admitted to our Cardiology Department were recruited, who were diagnosed with $\mathrm{HFpEF} / \mathrm{HFmrEF}$ according to current guidelines (LVEF $>40 \%$, NYHA class I-IV, NT-proBNP $>300 \mathrm{pg} /$ $\mathrm{mL}$ ) [15]. All included patients who had developed symptoms in the first 24-48 hours upon admission. Subjects with coronary artery disease consisting of previously diagnosed MI or unstable angina, more than mild valvular heart disease, pulmonary disease with pulmonary hypertension, were excluded. Since high blood pressure (BP) was demonstrated to have an impact on MW indices
[9], patients with uncontrolled arterial hypertension were also excluded. All patients underwent primary percutaneous revascularization (PCI). Echocardiography was performed in the first 24-48 hours after revascularization by using STE and BP was measured at the beginning of the echocardiographic study. Patients with improper quality of the images were excluded.

\section{Echocardiographic analysis}

All echocardiography images were acquired on a Vivid E95 scanner (GE Healthcare, Horten, Norway) using a 2D matrix array transducer (M5S) and stored in Digital Imaging format (EchoPac BT13, GE Vingmed Ultrasound). The M5S probe was used to acquire the standard 2D apical long axis views (apical four chamber-, 2 chamber- and 3 chamber-view). Simpson's biplane LVEF and the indices of diastolic dysfunction (trans-mitral peak early (E) and late (A) diastolic velocities, average e' assessed with tissue Doppler imaging, maximum tricuspid regurgitation (TR) velocity and left atrial (LA) volume index) were calculated according to current recommendations [16].

STE parameters were obtained using the automated function-imaging (AFI) tool. Automated tracking of the myocardial motion was performed and the region of interest was adjusted if necessary. GLS was calculated as the average of all peak regional strain values in the 17 segments of the LV. MW was calculated by using the combination between LV strain and non-invasively estimated LV pressure curves. Since peak systolic LV pres-

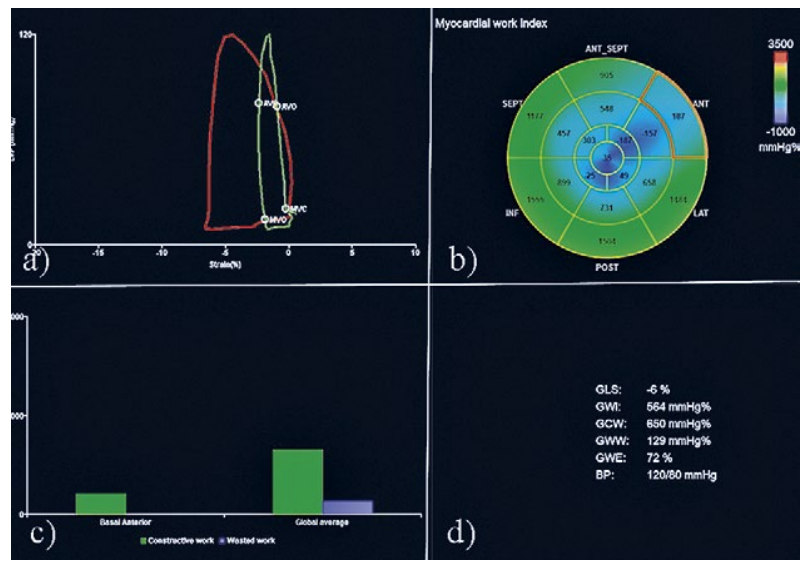

Fig 1. Measurement of MW by STE:a) LV pressure-strain loop (curve in green corresponds to the selected segment on the GWI bull's eye and shows a significantly reduced area compared to the curve in red, which corresponds to average work);b) Bull's eye of GWI (areas of negative work in blue and normal work in green);c) graphic representation of GCW and GWW;d) result after MW analysis. GCW, global constructive work; GWI, global work index; GWW; global wasted work; MW, myocardial work; STE, speckle-tracking echocardiography. 
sure is assumed to be equal to the peak arterial pressure, we recorded the systolic pressure measured by arterial cuff at the beginning of the echocardiographic study. The software constructed LV pressure strain-loops (PSL) according to valvular timing events, as shown in fig1. Aligning valvular event times synchronized strain and pressure data. The following MW parameters were obtained: global MW index (GWI) - defined as the work inside the area of the PSL, calculated from the mitral valve closure to its opening; global constructive $M W(G C W)$ - defined as the work which contributes to the LV ejection in systole, calculated as the sum of shortening during systole and lengthening during isovolumic relaxation; global wasted work $(G W W)$ - defined as the work of the LV which does not participate to LV ejection, calculated as the sum of lengthening in systole and shortening during isovolumic relaxation; global work efficiency (GWE) - calculated as the ratio of GCW and sum of GCW and GWW.

\section{Other measurements}

Venous samples were collected in the first 24-48 hours after percutaneous revascularization. For later analysis, plasma was collected in ethylene diamide-tetracetic (EDTA) vials and centrifuged at $4^{\circ} \mathrm{C}$ with $3000 \mathrm{rpm}$ in 10 minutes and afterwards stored at $-80^{\circ} \mathrm{Celsius}$. NT-proBNP was measured by sandwich enzyme-linked immunosorbent assay (NT-proBNP ELISA SK-1204 Biomedica Immunoassays, Vienna, Austria) according to manufacturer procedure and was expressed as $\mathrm{pg} / \mathrm{ml}$. Since current recommendations exclude acute HF if NT-proBNP $<300 \mathrm{mmHg} \%$, we only included patients with NT-proBNP above this value.

The maximum ST segment elevation and the percent of resolution (STR) at 90 minutes after PCI on electrocardiogram were recorded in all patients. The cut-off for complete STR was $\geq 70 \%$. The culprit vessel and the presence of multi-vascular coronary artery disease were recorded on angiography. Coronary blood flow patterns before and after primary PCI were evaluated using the TIMI flow grade.

\section{Intra- and inter-observer variability}

In order to test the intraobserver variability, ten patients were randomly selected and measurements were repeated. The inter-observer variability was tested by a second operator that was blinded to the results of the first operator.

\section{Statistical analysis}

Statistical analysis was performed using R Core Team 2019 (Vienna, Austria) and Microsoft Excel for Mac 2011. Continuous variables were expressed as mean \pm standard deviation (mean \pm SD) or median [IQR], according to the distribution of the data. Normality was tested using the Kolmogorov-Smirnov test. Correlations between variables were assessed using Spearman's correlation coefficient. Differences in echocardiographic parameters according to the mean value of GWI were calculated using t-tests if the distribution was normal and the Mann-Whitney U test otherwise. A p value of $<0.05$ was considered significant. Reproducibility was tested by calculating the variability with intra- and inter-observer correlation coefficients.

\section{Results}

\section{Patient characteristics}

Forty-nine patients (mean age $68 \pm 13$ years, 51\% men) fulfilled the inclusion criteria (Table I). There was a high burden of cardiovascular risk factors among the included patients. The most frequently associated risk factor was arterial hypertension, which was present in 37 $(83 \%)$ of the patients. Moreover, a high percent of the patients were overweight or obese $(n=29,60 \%)$. There was a higher proportion of STEMI $(n=33,67 \%)$ than NSTEMI among patients. The median of NT-proBNP values was of 800 [530-2060] pg/mL. Regarding symptomatology, $35(71 \%)$ patients accused dyspnea in the first 2 days of admission, but the majority was placed in NYHA I-II functional class ( $\mathrm{n}=31,63.2 \%)$.

\section{Echocardiographic analysis}

Patients with pressure-loaded LV had generally normal LV dimensions (2D linear measurements and volumes). All of the patients had a preserved or mildly reduced ejection fraction ( $>40 \%)$. Mean LVEF was $49.7 \pm 7.3 \%$. The systolic, diastolic and STE measurements are depicted in Table II.

Regarding the diastolic function, the most frequently impaired parameter was LA volume index, with 15 (31\%) patients showing elevated indexed volume. The majority of the patients demonstrated grade I or II diastolic dysfunction $(\mathrm{n}=48,98 \%)$. Even though LVEF was preserved or only mildly reduced in all patients, $90 \%$ had a GLS below $-14 \%$, but GWI was impaired in only $71 \%$ of patients (examples showed in fig2).

\section{Relationship between MW, other}

echocardiographic parameters and NT-proBNP

GWI showed a strong positive correlation to LVEF $(\mathrm{r}=0.69)$ and an inverse relationship to NT-proBNP $(\mathrm{r}=-$ $0.29)$. Moreover, we found strong correlations between MW and GLS. Both GWI and GWE showed an inverse relationship with GLS ( $\mathrm{r}=-0.81$ and $\mathrm{r}=-0.57)$.

Regarding diastolic dysfunction parameters, only LA volume index correlated to all STE parameters. E/A and E/e' were also correlated to GWI, but not to GLS, as seen in fig 3. Main correlations of GWI are depicted in fig 4 . 
Table I. General characteristics of the patients

\begin{tabular}{|c|c|}
\hline Variable & Values \\
\hline Age, years & $68 \pm 13$ \\
\hline Male sex, n (\%) & $25(51)$ \\
\hline BMI & $28 \pm 4.2$ \\
\hline Smoking, n (\%) & $12(25)$ \\
\hline $\begin{array}{l}\text { Arterial hypertension, } \mathrm{n}(\%) \\
\text { Grade I } \\
\text { Grade II } \\
\text { Grade III }\end{array}$ & $\begin{array}{l}37(83) \\
3(6) \\
22(45) \\
16(32)\end{array}$ \\
\hline Diabetes, n (\%) & $11(30)$ \\
\hline Dyslipidemia, n (\%) & $11(30)$ \\
\hline $\begin{array}{l}\text { Symptoms, } \mathrm{n}(\%) \\
\text { Dyspnea } \\
\text { Angina } \\
\text { Dyspnea and angina } \\
\text { Other symptoms }\end{array}$ & $\begin{array}{l}35(71) \\
39(79) \\
15(30) \\
5(10)\end{array}$ \\
\hline $\begin{array}{l}\text { NYHA functional class, n (\%) } \\
\text { Grade I-II } \\
\text { Grade III-IV }\end{array}$ & $\begin{array}{l}31(63.2) \\
18(36.8)\end{array}$ \\
\hline Biological data & \\
\hline NT-proBNP & $800[530-2060]$ \\
\hline Troponin & $0.3[0.1-1.1]$ \\
\hline Total cholesterol & $186 \pm 53$ \\
\hline LDL-cholesterol & $112 \pm 12$ \\
\hline HDL-cholesterol & $44 \pm 12$ \\
\hline Triglycerides & $143 \pm 80$ \\
\hline Creatinine & $0.9[0.7-1.1]$ \\
\hline Glucose & $141 \pm 60$ \\
\hline STEMI/ NSTEMI, n (\%) & $33(67) / 15(33)$ \\
\hline $\begin{array}{l}\text { Systolic blood pressure } \\
\text { on echocardiography, } \mathrm{mmHg}\end{array}$ & $131 \pm 25$ \\
\hline $\begin{array}{l}\text { Diastolic blood pressure } \\
\quad \text { on echocardiography, } \mathrm{mmHg}\end{array}$ & $73 \pm 16$ \\
\hline Heart rate on echocardiography, bpm & $79 \pm 20$ \\
\hline Maximum ST segment elevation, $\mathrm{mm}$ & $1.5 \pm 1.7$ \\
\hline $\begin{array}{l}\text { ST segment resolution after PCI, n (\%) } \\
\text { Complete / } \\
\text { incomplete }\end{array}$ & $\begin{array}{l}29(59.2) / \\
20(40.8)\end{array}$ \\
\hline $\begin{array}{l}\text { Culprit vessel, n (\%) } \\
\text { LAD / CX / } \\
\text { RCA }\end{array}$ & $\begin{array}{l}22(45) / 9(18) / \\
18(37)\end{array}$ \\
\hline $\begin{array}{l}\text { TIMI flow grade after AMI, n (\%) } \\
\begin{array}{l}3 \\
0-2\end{array}\end{array}$ & $\begin{array}{l}33(67) \\
12(33)\end{array}$ \\
\hline $\begin{array}{l}\text { Life-threatening conditions, n }(\%) \\
\text { Hypotension } \\
\text { Ventricular arrhythmias } \\
\text { Cardiac arrest } \\
\text { Cardiac death }\end{array}$ & $\begin{array}{l}2(4) \\
7(14) \\
4(8) \\
4(8)\end{array}$ \\
\hline
\end{tabular}

AMI, acute myocardial infarction; BMI, body mass index; CX, circumflex coronary artery; LAD, left anterior descending coronary artery; NSTEMI, non-ST-segment elevation acute myocardial infarction; RCA, right coronary artery; STEMI, ST-segment elevation acute myocardial infarction.
Since we aimed to evaluate the impact of pressure overload on LVEF, NT-proBNP and diastolic function parameters, we divided the patients in two groups according to the median values of GWI (1057 $\mathrm{mmHg} \%$ ), as shown in Table III. We found that subjects with GWE values below the median showed three significantly more

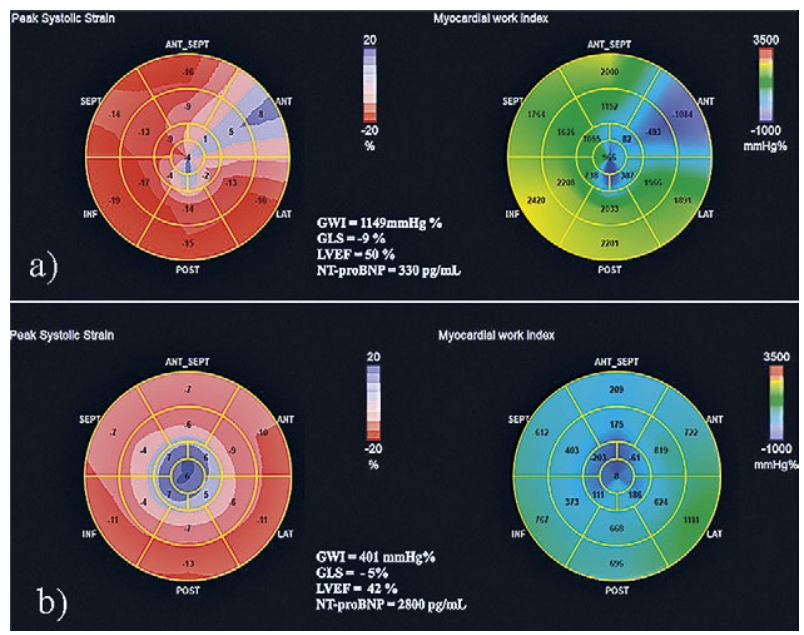

Fig 2. Bull's eye of GLS (on the left) and bull's eye of GWI (on the right) from two patients with anterior AMI. Note that although both GLS and GWI were impaired in both patients, patient a) showed higher GWI and lower NT-proBNP and patient b) significantly impaired GWI and high NT-proBNP.GLS, global longitudinal strain; GWI, global work index

\begin{tabular}{|c|c|c|c|c|c|c|c|c|c|c|}
\hline GLS & 1 & -0.81 & -0.57 & -0.63 & 0.31 & NS & NS & NS & NS & 0.34 \\
\hline GWI & -0.81 & 1 & 0.61 & 0.69 & -0.29 & NS & -0.39 & -0.23 & NS & -0.29 \\
\hline GWE & -0.57 & 0.61 & 1 & 0.40 & NS & NS & NS & NS & NS & -0.30 \\
\hline LVEF & -0.63 & 0.69 & 0.40 & 1 & -0.30 & NS & NS & -0.46 & -0.28 & 0.27 \\
\hline NT-proBNP & 0.31 & -0.29 & NS & -0.30 & 1 & NS & NS & NS & NS & NS \\
\hline E & NS & NS & NS & NS & NS & 1 & 0.21 & 0.55 & NS & NS \\
\hline $\mathrm{E} / \mathrm{A}$ & 0.24 & -0.39 & NS & NS & NS & 0.21 & 1 & NS & 0.27 & 0.26 \\
\hline $\mathrm{E} / \mathrm{e}^{\prime}$ & 0.21 & -0.33 & NS & -0.46 & NS & 0.55 & NS & 1 & 0.24 & 0.21 \\
\hline TR velocity & NS & NS & NS & -0.28 & NS & NS & 0.27 & 0.24 & 1 & 0.47 \\
\hline LA vol. index & 0.34 & -0.29 & $\mid-0.30$ & 0.27 & NS & NS & 0.26 & 0.21 & 0.47 & 1 \\
\hline & 3 & 芯 & $\sum_{0}^{1}$ & $\sum_{3}^{5}$ & 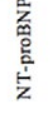 & 10 & 约 & 商 & 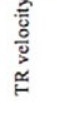 & 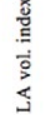 \\
\hline
\end{tabular}

Fig 3. Correlations between speckle-tracking parameters, diastolic function parameters, NT-proBNP and LVEF. GLS, global longitudinal strain; GWE, global work efficiency; GWI, global work index; LA, Left atrium; LVEF, left ventricular ejection fraction; TR, tricuspid regurgitation. 
Table II. Echocardiographic data of the patients

\begin{tabular}{|c|c|c|}
\hline & Echocardiographic variable & Values \\
\hline \multirow[t]{2}{*}{ Systolic function } & LVEF, \% & $49.7 \pm 7.3$ \\
\hline & LVEDD, mm & $48 \pm 5$ \\
\hline \multirow[t]{3}{*}{ LV diameters and volumes } & LVESD, mm & $34 \pm 5$ \\
\hline & LVEDV, $\mathrm{ml}$ & $108 \pm 32$ \\
\hline & LVESV, ml & $55 \pm 23$ \\
\hline \multirow[t]{21}{*}{ Diastolic function } & $\mathbf{E}, \mathrm{m} / \mathrm{s}$ & $0.65 \pm 0.16$ \\
\hline & $\leq 0.5 \mathrm{~m} / \mathrm{s}, \mathrm{n}(\%)$ & $12(24)$ \\
\hline & $>0.5 \mathrm{~m} / \mathrm{s}, \mathrm{n}(\%)$ & $37(76)$ \\
\hline & $\mathbf{E} / \mathbf{A}$ & $1.24 \pm 0.5$ \\
\hline & $\leq 0.8, \mathrm{n}(\%)$ & $38(77)$ \\
\hline & $>0.8, \mathrm{n}(\%)$ & $10(20)$ \\
\hline & $\geq 2, \mathrm{n}(\%)$ & $1(3)$ \\
\hline & $\mathbf{E} / \mathbf{e}^{\prime}$ & $9.5 \pm 4.6$ \\
\hline & $\leq 14, \mathrm{n}(\%)$ & $42(86)$ \\
\hline & $>14, \mathrm{n}(\%)$ & $7(14)$ \\
\hline & LA volume index, $\mathrm{ml} / \mathrm{m}^{2}$ & $29 \pm 6.3$ \\
\hline & $\leq 34 \mathrm{ml} / \mathrm{m}^{2}, \mathrm{n}(\%)$ & $34(69)$ \\
\hline & $>34 \mathrm{ml} / \mathrm{m}^{2}, \mathrm{n}(\%)$ & $15(31)$ \\
\hline & TR velocity, m/s & $2.5 \pm 1$ \\
\hline & $\leq 2.8 \mathrm{~m} / \mathrm{s}, \mathrm{n}(\%)$ & $38(77)$ \\
\hline & $>2.8 \mathrm{~m} / \mathrm{s}, \mathrm{n}(\%)$ & $11(23)$ \\
\hline & Grade of diastolic dysfunction & \\
\hline & Grade 1 & $27(55)$ \\
\hline & Grade 2 & $8(16)$ \\
\hline & Grade 1 or 2 ( $1 / 3$ criteria) & $13(27)$ \\
\hline & Grade 3 & $1(2)$ \\
\hline \multirow[t]{9}{*}{ STE measurements } & GLS, \% & $-10.4 \pm 3.3$ \\
\hline & $\geq-14 \%$ & $5(10)$ \\
\hline & $<-14 \%$ & $44(90)$ \\
\hline & GWI, mmHg\% & $1057 \pm 338$ \\
\hline & $\geq 1270 \mathrm{mmHg} \%$ & $14(29)$ \\
\hline & $<1270 \mathrm{mmHg} \%$ & $35(71)$ \\
\hline & GWE, \% & $82 \pm 8.3$ \\
\hline & $\geq 90 \%$ & $11(22)$ \\
\hline & $<90 \%$ & $38(78)$ \\
\hline
\end{tabular}

GLS, global longitudinal strain; GWE, global work efficiency; GWI, global work index; LA. Left atrium; LV, left ventricle; LVEDD, left ventricular end-diastolic diameter; LVEV, left ventricular end-diastolic volume; LVESD; left ventricular end-systolic volume; LVESV; left ventricular end-systolic volume; LVEF, left ventricular ejection fraction; TR, tricuspid regurgitation.
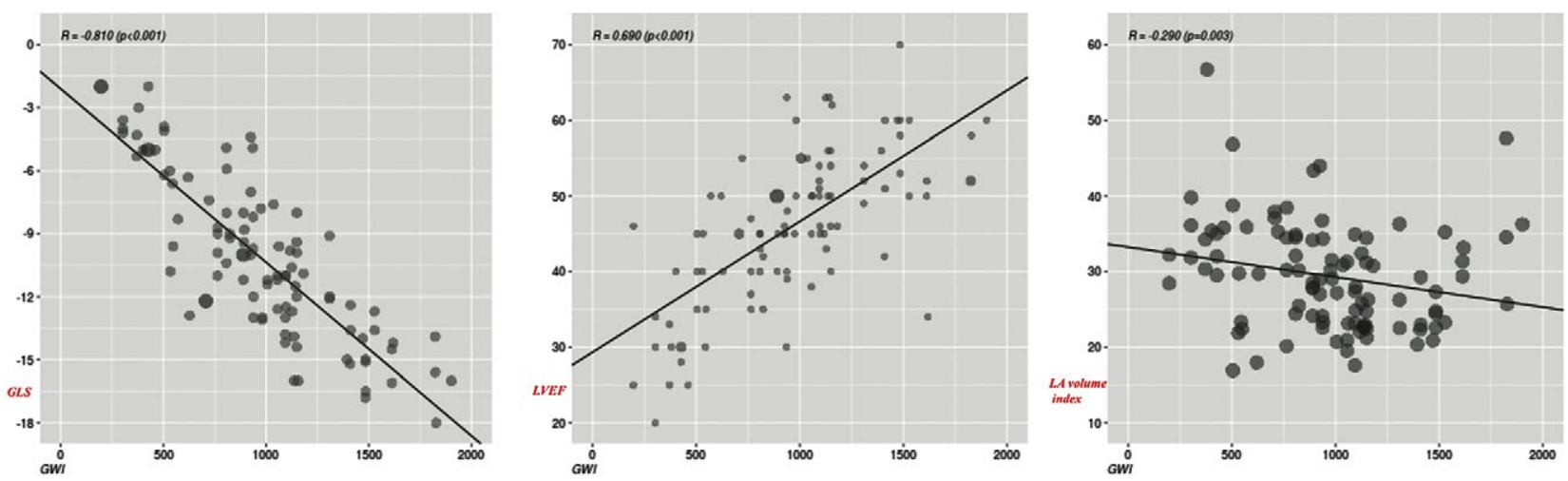

Fig 4. Main correlations of GWI. GLS, global longitudinal strain; GWI, global work index; LA, Left atrium; LVEF, left ventricular ejection fraction. 
Table III. Differences between LVEF, NT-proBNP and diastolic dysfunction parameters in patients with GWI below and above the median of $1057 \mathrm{mmHg} \%$

\begin{tabular}{llll}
\hline Variables & GWI $\leq \mathbf{1 0 5 7} \mathbf{~ m m H g \%}$ & GWI $>$ 1057 $\mathbf{~ m m H o \%}$ & p \\
\hline LVEF, \% & $46 \pm 6$ & $53 \pm 7$ & $<\mathbf{0 . 0 0 1 *}$ \\
NT-proBNP, pg/mL & $820[590-2550]$ & $707[460-1335]$ & 0.17 \\
E, m/s & $0.7 \pm 0.2$ & $0.6 \pm 0.14$ & 0.16 \\
E/A & $1.4 \pm 0.6$ & $1.1 \pm 0.3$ & $\mathbf{0 . 0 3 *}$ \\
E/e & $11 \pm 6$ & $8 \pm 3$ & $\mathbf{0 . 0 1 *}$ \\
LA volume index $\left(\mathrm{mL} / \mathrm{m}^{2}\right)$ & $32 \pm 7$ & $26 \pm 5$ & $<\mathbf{0 . 0 0 1}$ \\
TR velocity $(\mathrm{m} / \mathrm{s})$ & $2.7 \pm 1.4$ & $2.3 \pm 0.6$ & 0.08 \\
\hline
\end{tabular}

GWI, global work index; LA, left atrium; LVEF, left ventricular ejection fraction; TR, tricuspid regurgitation.

impaired diastolic parameters $(\mathrm{p}<0.001$ for LA volume index, $\mathrm{p}=0.01$ for $\mathrm{E} / \mathrm{e}$ ' and $\mathrm{p}=0.3$ for $\mathrm{E} / \mathrm{A})$. NT-proBNP values also showed a tendency to higher values in the group with GWI below the median (820 [590-2550] vs 707 [460-1335] mmHg\%), but without statistical significance $(\mathrm{p}=0.17)$.

\section{Reproducibility}

A total of 10 patients were randomly selected in order to assess the reproducibility of the STE measurements. Intra- and inter-observer interclass correlation coefficients for GLS, GWI and GWE were of 0.93 and 0.95 , 0.94 and $0.92,0.96$ and 0.92 , showing a high agreement between measurements.

\section{Discussion}

The main results of our study were: 1) both GWI and GLS were decreased in the majority of the patients; 2) despite abnormal GLS, there was a proportion of patients with normal GWI; 3) GWI demonstrated a strong relationship to LVEF and a moderate correlation to NTproBNP; 4) GWI showed correlations to diastolic dysfunction parameters.

\section{Relationship between MW and GLS}

In patients with AMI, reduction in systolic function depends on both contractile properties and variations in load in ischemic segments. In our group, although LVEF was preserved or only mildly reduced and LV 2D dimensions were generally normal, both GWI and GLS were impaired in almost all subjects. As there are no currently known cutoff values for GWI, we used as reference those of Manganaro et al [17], which reported a median of $1896 \pm 308 \mathrm{mmHg} \%$ for GWI (lowest normal value of $1270 \mathrm{mmHg} \%$ ) and a median of $96 \%$ for GWE (lowest expected value of $90 \%$ ) in a healthy population. However, another study found significantly lower GWI values of $916 \pm 452 \mathrm{mmHg} \%$ in patients with ischemic cardiomyopathy [9], while Hedwig et al reported a median of GWI of $653 \pm 379 \mathrm{mmHg} \%$ in patients with HF [14]. The reduced values of MW indices we found in patients with AMI and HFpEF/ HFmrEF reflect the impairment of LV performance and might contribute to early LV remodeling.

In our study, the proportion of patients with impaired GLS (90\% of the patients showed GLS values $\leq-14 \%)$ was higher than of those with impaired MW (71\% percent of the patients demonstrated GWI $<1270 \mathrm{mmHg}$ ). In patients with pressure-loaded LV, increased afterload can decrease strain, even if MW is normal. This might lead to the misinterpretation of decreased contractility, when it is actually an increase in afterload $[9,10,18]$. However, Chan et al. also found a significant decrease in the values of GWI (916 $\pm 452 \mathrm{mmHg} \%$ ) along with GLS (9.4 $\pm 4.1 \%)$ in patients with ischemic cardiomyopathy, showing that both MW indices and GLS are impaired in ischemic heart disease [9]. This might be the result of higher levels of wasted work and lower levels of efficient work in patients with AMI.

As an association was expected, GWI and GLS showed a strong inverse relationship $(\mathrm{r}=-0.81)$. Manganaro et al also found a significant association between the two parameters $(\mathrm{r}=-0.51)$ in a healthy population [19], which could be explained by the important contribution of deformation in the generation of MW.

MW might be underestimated in dilated ventricles due to higher wall stress [9]. Thus, we highlight that MW might be a promising tool in the risk assessment of remodeling in patients with AMI and HFpEF, who show altered LV performance at the early stage of the disease, when LVEF is still preserved and LV in not yet dilated. MW parameters demonstrated excellent intra- and interobserver reproducibility, with interclass correlation coefficients of $>91 \%$. Variability was similar to that reported in other studies $[9,17]$.

\section{Relationship between MW and diastolic dysfunction parameters}

The diagnosis of HFpEF or HFmrEF is more challenging than of HF with reduced ejection fraction (HFrEF), as 
demonstrated in previous studies $[15,16]$. These patients have increased filling pressures, even in the presence of a LV with normal dimensions. In our study, the majority of the patients demonstrated grade I/II diastolic dysfunction, while LA volume index was the most frequently impaired diastolic dysfunction parameter, confirming that in the course of HFpEF progression, starting with elevation of the LV end-diastolic pressure, LA function is also impaired $[16,20]$. However, GWI was impaired in more patients than diastolic parameters alone were $(71 \%$ vs. $31 \%$ for LA volume index).

As there are no currently known cutoff values for GWI, we divided the subjects in groups according to the median of GWI (1057 $\mathrm{mmHg} \%)$. This was done in order to find differences in the values of already known parameters, which might occur in pressure-loaded ventricles. We found that subjects with more reduced GWI showed three significantly more impaired diastolic dysfunction parameters (LA volume index, E/A, E/e'). Moreover, GWI showed a relationship to LA volume index, E/A and $\mathrm{E} / \mathrm{e}$, demonstrating the connection between MW and LV filling pressures. Chan et al also found reduced E/e' in patients with ischemic cardiomyopathy and reduced GWI [9], while Cauwenbergs et al demonstrated that GWI decreased with elevated LA volume index and reduced E/e' ratio in a healthy population [3]. These results overall demonstrate that increased LV end-diastolic pressure and LA stiffness occurring in the early stages of $\mathrm{HFpEF} /$ HFmrEF are associated with reduced MW.

\section{Relationship between MW, LVEF, NT-proBNP and symptoms}

The importance of LVEF in the prediction of HF is well known [15]. GWI demonstrated a strong relationship with LVEF. Other studies also found significantly lower LVEF in patients with reduced GWI and ischemic cardiomyopathy [9] or HF [14].

NT-proBNP is a well-known predictor of outcome of HFrEF, but its role in the prognosis of patients with HFpEF and HFmrEF remains controversial. However, it remains a marker of ventricular function and remodeling [15]. Although the correlation between GWI and NTproBNP was only moderate, NT-proBNP values showed a tendency to being higher in the group with GWI below the median. Hedwig et al found significantly more elevated NT-proBNP in patients with reduced GWI, demonstrating the connection between MW and myocardial stress, but the majority of the patients in that study had a reduced LVEF [14].

Some patients in our study showed incomplete ST segment resolution after PCI or reduced TIMI flow grade. Therefore, the persistence of dyspnea in these patients after revascularization could be related to increased myocardial wall stress, a more severe extension of AMI, diminished myocardial reperfusion and in some cases as an adverse effect of antiplatelet therapy (ticagrelor).

\section{Associated comorbidities in patients with $\mathrm{HFpEF}$}

\section{and HFmrEF after AMI}

There was a high burden of cardiovascular risk factors among patients with HFpEF and HFmrEF. Arterial hypertension ( $83 \%$ of the patients) and obesity $(60 \%$ of the patients) were most frequently associated. It was demonstrated by previous studies that both of these cardiovascular risk factors are associated with an increased risk of developing HF $[15,21,22]$. Moreover, obesity is more commonly associated with HFpEF than HFrEF [15,21].

\section{Limitations and future directions}

Calculation of global GWI does not take into account the complex geometry of the LV, which particularly occurs in patients with AMI due to wall motion anomalies. This might be a limitation of our study. Moreover, cardiac magnetic resonance imaging (MRI) remains the gold standard in the evaluation of infarct size [23]. Future studies should therefore include three-dimensional measurements of the LV dimensions, assessment of extension of myocardial infarction by cardiac MRI and larger cohorts of patients. Furthermore, as there is no currently known cutoff value for GWI to predict the occurrence of HF or remodeling, future studies should focus on finding this limit.

\section{Conclusions}

In patients with AMI, impairment of the LV systolic function depends on both contractile properties and variations in load in the ischemic segments. Non-invasive pressure-strain loops, which incorporate both deformation and afterload, bring further information to LVEF and GLS in the quantification of LV performance at the early stages of $\mathrm{HFpEF} / \mathrm{HFmrEF}$.

Acknowledgements: This research was funded by "Iuliu Haţieganu" University of Medicine and Pharmacy, Deptartment of Cardiology, Cluj-Napoca, Romania, grant number 1529/66/18.01.2019.

\section{Conflicts of interest: none}

\section{References}

1. Borlaug BA. The pathophysiology of heart failure with preserved ejection fraction. Nat Rev Cardiol 2014;11:507-515.

2. Cauwenberghs N, Tabassian M, Thijs L, et al. Area of the pressure-strain loop during ejection as non-invasive index of left ventricular performance: a population study. Cardiovasc Ultrasound 2019;17:15. 
3. Cauwenberghs N, Knez J, D'hooge J, et al. Longitudinal changes in $\mathrm{LV}$ structure and diastolic function in relation to arterial properties in general population. JACC Cardiovasc Imaging 2017;10:1307-1416.

4. Cauwenberghs N, Knez J, Tikhonoff V, et al. Doppler indexes of left ventricular systolic and diastolic function in relation to the arterial stiffness in a general population. $\mathrm{J}$ Hypertens 2016;34:762-771.

5. Konstam MA, Abboud FM. Ejection fraction: misunderstood and overrated (changing the paradigm in categorizing heart failure). Circulation 2017;135:717-719.

6. Tomoaia R, Beyer RS, Simu G, Serban AM, Pop D. Understanding the role of echocardiography in remodeling after acute myocardial infarction and development of heart failure with preserved ejection fraction - new aspects of interest. Med Ultrason 2019;21:69-76.

7. Ersboll M, Valeur N, Mogensen UM, et al. Prediction of all-cause mortality and heart failure admissions from global left ventricular longitudinal strain in patients with acute myocardial infarction and preserved left ventricular ejection fraction. J Am Coll Cardiol 2013;61:2365-2373.

8. Mor-Avi V, Lang RM, Badano LP, et al. Current and evolving echocardiographic techniques for the quantitative evaluation of cardiac mechanics: ASE/EAE consensus statement on methodology and indications endorsed by the Japanese Society of Echocardiography. Eur J Echocardiogr 2011;12:167-205.

9. Chan J, Edwards NFA, Khandheria BK, et al. A new approach to assess myocardial work by non-invasive left ventricular pressure-strain relations in hypertension and dilated cardiomyopathy. Eur Heart J Cardiovase Imaging. 2019;20:31-39.

10. Hubert A, Le Rolle V, Leclercq C, et al. Estimation of myocardial work from pressure-strain loops analysis: an experimental evaluation. Eur Heart J Cardiovasc Imaging 2018;19:1372-1379.

11. Russell K, Eriksen M, Aaberge L, et al. A novel clinical method for quantification of regional left ventricular pressurestrain loop area: a non-invasive index of myocardial work. Eur Heart J 2012;33:724-733.

12. Russell K, Eriksen M, Aaberge L, et al. Assessment of wasted myocardial work: a novel method to quantify energy loss due to uncoordinated left ventricular contractions. Am J Physiol Heart Circ Physiol 2013;305:H996H1003.
13. Boe E, Russell K, Eek C, et al. Non-invasive myocardial work index identifies acute coronary occlusion in patients with non-ST-segment elevation-acute coronary syndrome. Eur Heart J Cardiovasc Imaging 2015;16:1247-1255.

14. Hedwig F, Soltani S, Stein J, et al. Global work index correlates with established prognostic parameters of heart failure. Echocardiography 2020;37:412-420.

15. Ponikowski P, Voors AA, Anker SD, et al. 2016 ESC Guidelines for the diagnosis and treatment of acute and chronic heart failure: The Task Force for the diagnosis and treatment of acute and chronic heart failure of the European Society of Cardiology (ESC) Developed with the special contribution of the Heart Failure Association (HFA) of the ESC. Eur Heart J 2016;37:2129-2200.

16. Nagueh SF, Smiseth OA, Appleton CP, et al. Recommendations for the Evaluation of Left Ventricular Diastolic Function by Echocardiography: An Update from the American Society of Echocardiography and the European Association of Cardiovascular Imaging. Eur Heart J Cardiovasc Imaging 2016;17:1321-1360.

17. Manganaro R, Marchetta S, Dulgheru R, et al. Echocardiographic reference ranges for normal non-invasive myocardial work indices: results from the EACVI NORRE study. Eur Heart J Cardiovasc Imaging 2019;20:582-590.

18. Skulstad H, Edvardsen T, Urheim S, et al. Postsystolic shortening in ischemic myocardium: active contraction or passive recoil? Circulation 2002;106:718-724.

19. Manganaro R, Marchetta S, Dulgheru R, et al. Correlation between non-invasive myocardial work indices and main parameters of systolic and diastolic function: results from the EACVI NORRE study. Eur Heart J Cardiovasc Imaging 2020;21:533-541.

20. Kuppahally SS, Akoum N, Burgon NS, et al. Left atrial strain and strain rate in patients with paroxysmal and persistent atrial fibrillation: relationship to left atrial structural remodeling detected by delayed-enhancement MRI. Circ Cardiovasc Imaging 2010;3:231-239.

21. Kenchaiah S, Evans JC, Levy D, et al. Obesity and the risk of heart failure. N Engl J Med 2002;347:305-313.

22. Dădârlat-Pop A, Sitar-Tăut A, Zdrenghea D, et al. Profile of Obesity and Comorbidities in Elderly Patients with Heart Failure. Clin Interv Aging 2020;15:547-556.

23. Reindl M, Eitel I, Reinstadler SJ. Role of Cardiac Magnetic Resonance to Improve Risk Prediction following Acute STelevation Myocardial Infarction. J Clin Med 2020;9:1041. 